\title{
VALIDACIÓN DE UN INSTRUMENTO PARA LA VALORACIÓN DE LA GLOBALIDAD DE LOS ESTÁNDARES CURRICULARES: ESTUDIO DE CASO DEL CURRÍCULO DE EDUCACIÓN FÍSICA EN ANDALUCÍA
}

Validation of an instrument for the assessment of the globality of curricular standards: study of the case of the curriculum of physical education in Andalucia

Validação de um instrumento para a valoração da globalidade dos padrões curriculares: estudo de caso do currículo de educação física na Andaluzia

\section{Fernando Manuel Otero-Saborido (1)}

Francisco Javier Vázquez-Ramos (1)

(1) Universidad Pablo de Olavide, Sevilla, España. Teléfono: +34 954977513 Correo electrónico: fmotero@upo.es

\section{Resumen}

Los currículos por competencias han abanderado la legislación educativa en los últimos años. No obstante, se aprecia una contradicción entre la perspectiva globalista de estos currículos y la excesiva estandarización y operativización de los referentes evaluativos. Por ello, el objetivo de este trabajo fue diseñar y validar un instrumento que valorara el grado de globalidad de los referentes evaluativos tomando como muestra el currículo de Educación Física de Primaria en Andalucía. Fruto de la validación de contenido, se diseñó un instrumento con tres dimensiones: conceptual, motriz y socio-actitudinal. Los resultados obtenidos muestran una alta fiabilidad del instrumento medida a través de la concordancia del índice Kappa de Cohen (Conceptual: 0.947; Motriz: 0,952; Socioactitudinal: 0.954).

Palabras clave: Competencias; evaluación formativa; criterios de evaluación

\begin{abstract}
The curricula for competences have championed the educational legislation in recent years. However, there is a contradiction between the globalist perspective of these curricula and the excessive standardization and operationalization of the evaluative referents. Therefore, the objective of this work was to design and validate an instrument that will assess the degree of globality of the evaluative referents, taking reference to the curriculum of Primary Education in Andalusia. As a result of content validation, an instrument was designed with three dimensions: conceptual, motor and socio-attitudinal.


The results obtained show a high reliability of the instrument measured through the concordance of Cohen's Kappa index (Conceptual: 0.947, Motor: 0.952, Socioattitudinal: 0.954).

Keywords: Competencies; formative assessment; assessment indicators

\section{Resumo}

Os currículos por competências têm defendido a legislação educativa nos últimos anos. No entanto, observa-se uma contradição entre a perspetiva global destes currículos e a excessiva padronização e operacionalização das referências avaliativas. Nesse sentido, o objetivo deste trabalho foi desenhar e validar um instrumento que valorasse o grau de globalidade das referências avaliativas, tendo como referência o currículo de Educação Física de Primária na Andaluzia. Como resultado da validação de conteúdo, foi desenhado um instrumento com três dimensões: concetual, motora e sócio atitudinal. Os resultados obtidos mostram uma alta fiabilidade do instrumento, medida através da concordância do índice Kappa de Cohen (Concetual: 0,947, Motor: 0,952, Socio atitudinal: 0,954).

Palavras-chave: Competências; avaliação formativa; critérios de avaliação

\section{Introducción}

La evaluación es uno de los aspectos más controvertidos en las instituciones educativas debido a las connotaciones éticas e ideológicas que emanan como fenómeno dentro del proceso de enseñanza aprendizaje (Santos-Guerra, 2003). Tras la aparición de los currículos por competencias, esta importancia de la evaluación ha sido recogida por las diferentes normativas curriculares tanto a nivel nacional (Real Decreto 126/2014, de 28 de febrero, por el que se establece el currículo básico de la Educación Primaria) como en los diferentes currículos autonómicos. Un estudio de los 17 currículos autonómicos de Educación Física en Primaria refleja la involución pedagógica en materia de evaluación con un aumento de la conductualización y operativización de la evaluación (Otero-Saborido \& Vázquez-Ramos, 2019). Una primera conclusión del trabajo anterior refleja una regresión del currículo hacia la medición, que no evaluación. Por ello, tras es este primer estudio exploratorio realizado sobre los estándares evaluativos de los 17 currículos de EF, y partiendo de la hipótesis generada por Otero-Saborido y Vázquez- 
Ramos (2019) que un aumento del número de estándares pudiera restar globalidad y, por ende, carácter educativo a la evaluación, el objetivo del siguiente trabajo fue diseñar y validar un instrumento que valorara el grado de globalidad de los referentes evaluativos. En este trabajo, se tomó como muestra el caso del currículo de EF en Primaria de Andalucía (Orden 17 de marzo de 2015) por poseer un reducido número de estándares evaluativos y orientar al profesorado con ejemplificaciones concretas sobre estrategias metodológicas y procesuales de evaluación en los diferentes referentes evaluativos.

\section{Método}

El proceso de validación consistió en dos fases. En primer lugar, una validación de contenido y, en segundo lugar, se aplicó un procedimiento de fiabilidad usando el índice de Kappa de Cohen desde dos puntos de vista. En primera instancia, desde la fiabilidad de dos inter-observadores y, en segundo lugar, desde la fiabilidad temporal. Por último, se halló el coeficiente de eficiencia evaluativa entendido como el cociente originado de la división entre la puntuación de globalidad de los estándares de todo el currículo y la máxima puntuación posible.

En el caso de la validación de contenido, para la determinación de las dimensiones que valorarían el grado de globalidad los dos expertos revisaron la literatura sobre ese aspecto tanto general como específica del área de EF (Delors, 1996; López-Pastor, 1999; Pérez-Pueyo, Julián-Clemente \& López-Pastor, 2011; Otero-Saborido, 2008). Tras dos circulaciones utilizando una muestra de referentes evaluativos del currículo de Andalucía, las dimensiones seleccionadas para valorar la globalidad de los estándares fueron: cognitivo, motriz y socio-actitudinal.

Tras la definición y ejemplificación de los criterios señalados, se dedicaron dos sesiones de entrenamiento de una hora para que los expertos unificaran criterios y se entrenaran en la asignación de dimensiones a cada estándar. Posteriormente, y de forma separada, los expertos valoraron los 44 estándares evaluativos asignando 1 punto en el caso de reconocer la presencia de esa dimensión o 0 puntos en el caso contrario. De esta categorización se obtiene la puntuación de globalidad. Pasados quince días de la primera valoración, los expertos realización una segunda categorización del instrumento con objeto de hallar la fiabilidad temporal. 


\section{Resultados y discusión}

Los resultados muestran una confiabilidad perfecta, medida a través del coeficiente Kappa de Cohen, en las tres dimensiones del instrumento (Conceptual: 1.00; Motriz: 1,00; Socio-actitudinal: 1.00) con altos niveles de significación para las tres categorías ( $\mathrm{p}=0.000)$ (Tabla 1). Así mismo, según la clasificación de Landis y Koch (1977), el índice de Kappa de Cohen puede considerarse con una concordancia 'perfecta'.

Tabla 1.

Confiablidad interobservadores en las tres dimensiones que valoraran el grado de globalidad de los referentes evaluativos.

\begin{tabular}{lccc}
\hline & N & Coeficiente de Kappa & Significación \\
\hline Conceptual & 44 & 1,00 &, 000 \\
Motriz & 44 & 1,00 &, 000 \\
Socio-actitudinal & 44 & 1,00 &, 000 \\
\hline
\end{tabular}

Por último, la fiabilidad temporal obtuvo resultados similares de casi perfecta concordancia (Tabla 2) a los obtenidos en la medición anterior. El coeficiente de eficiencia evaluativa obtenido tras dividir la puntuación de globalidad obtenida y la máxima posible fue de 0,55 . A pesar de alta prestación de fiabilidad del instrumento es necesario discutir varias apreciaciones. Una primera herramienta original barajó la posibilidad de las 4 o 5 dimensiones (Delors, 1996). Sin embargo, esto presentaba dos sesgos, uno de carácter práctico y otro pedagógico.

Tabla 2.

Confiablidad temporal en las tres dimensiones que valoraran el grado de globalidad de los referentes evaluativos

\begin{tabular}{llcc}
\hline & N & Coeficiente de Kappa & Significación \\
\hline Conceptual & 44 &, 947 &, 000 \\
Motriz & 44 &, 952 &, 000 \\
Socio-actitudinal & 44 &, 954 &, 000 \\
\hline
\end{tabular}

En primer lugar, dada la intención práctica de esta herramienta para valorar el carácter integrador de los estándares de diferentes currículos, en las primeras rondas entre los expertos se comprobó que la fiabilidad se veía afectada y que difícilmente los expertos pudieran categorizar sin ambigüedad cuando el instrumento presentaba más de tres categorías. Por otra parte, compartimentar en exceso el aprendizaje y la conducta discente contradecía la perspectiva globalista de un currículo con vocación educativa. 
Por ello, tal y como señala López-Pastor (1999), si evaluar 3 dimensiones al mismo tiempo ya supone un auténtico reto para la EF, no parecía conveniente una discriminación excesiva de las dimensiones del discente de EF. Como colofón, si bien no se hallaron antecedentes bibliográficos que aporten herramientas capaces de precisar la globalidad de los referentes evaluativos, son dignos de reseña los antecedentes literarios sobre un currículo evaluativo, abierto y orientado al aprendizaje que no se limita a medir conductas (Apple, 1987; Gimeno-Sacristán, 1988, Stenhouse, 1987)

\section{Conclusiones}

Se ha diseñado un instrumento que se muestra válido y fiable en su primera fase exploratoria para valorar el grado de globalidad de los estándares de evaluación del currículo de EF en Primaria en el caso de Andalucía. Futuros trabajos deberán explorar mecanismos comparativos sobre el coeficiente de eficiencia evaluativa entre los diferentes currículos autonómicos. El objetivo no es otro que continuar con la línea de trabajos anteriores que generaron la hipótesis de que un mayor número de estándares evaluativos de los currículos de EF en las diferentes comunidades están contribuyendo a subrayar la medición, a anular el papel investigador de los actores educativos y poner el foco exclusivo en lo motriz en detrimento del valor educativo de una evaluación integral.

\section{Referencias}

Apple, M. (1987). Ideología y currículum. Madrid: Akal.

Delors, J. (1996). Los cuatro pilares de la educación. En "La educación encierra un tesoro”. Informe a la UNESCO de la Comisión internacional sobre la educación para el siglo XXI, (pp. 91-103). Madrid: Santillana/UNESCO.

Gimeno-Sacristán, J. (1989). El currículum: una reflexión sobre la práctica. Morata: Madrid.

Landis, J. R. \& Koch, G.G. (1977). The Measurement of Observer Agreement for Categorical Data. Biometrics, 33(1), 159-174

López-Pastor, V. M. (1999). Prácticas de evaluación en educación física: Estudio de casos en Primaria, Secundaria y formación del profesorado (Tesis doctoral inédita). Valladolid: Secretariado de Publicaciones de la Universidad de Valladolid. 
Orden de 17 de marzo de 2015, por la que se desarrolla el currículo correspondiente a la Educación Primaria en Andalucía (BOJA núm. 60 de 27/03/2015)

Otero, F.M. (2008). IV Congress Nacional y XXV Congreso Internacional de Educación Física: Hacia la concepción de las situaciones motrices globales en Educación Física: diseño y valoración. Córdoba.

Otero-Saborido, F.M. \& Vázquez-Ramos, F.J. (2019). La Evaluación Educativa en el Currículo LOMCE en Primaria: Análisis de los Currículos Autonómicos en Educación Física. Revista Iberoamericana de Evaluación Educativa, 12(1), 4758.

Pérez-Pueyo, A. Julián-Clemente, J.A. \& López-Pastor, V. (2011) Evaluación formativa y Compartida en el Espacio Europeo de Educación Superior En V. López-Pastor (2011). Evaluación formativa y Compartida en Educación Superior. Propuestas, técnicas, instrumentos y experiencias. (pp. 19-44). Madrid: Narcea.

Real Decreto 126/2014, de 28 de febrero, por el que se establece el currículo básico de la Educación Primaria (BOE núm. 52, 1/3/2014).

Santos, M.A. (2006). Una flecha en la diana: La Evaluación como Aprendizaje. Madrid: Narcea.

Stenhouse, L. (1987). La investigación como base de la enseñanza. Madrid: Morata. 